

\title{
Bayesian Analysis of RR Lyrae Luminosities and Kinematics
}

Thomas R. Jefferys, Thomas G. Barnes, Andrei Dambis, and William H. Jefferys

Citation: AIP Conference Proceedings 954, 315 (2007); doi: 10.1063/1.2821277

View online: http://dx.doi.org/10.1063/1.2821277

View Table of Contents: http://scitation.aip.org/content/aip/proceeding/aipcp/954?ver=pdfcov

Published by the AIP Publishing

\section{Articles you may be interested in}

A seasonal and heteroscedastic gamma model for hydrological time series: A Bayesian approach

AIP Conf. Proc. 1490, 97 (2012); 10.1063/1.4759593

RR Lyrae in LMC Globular Clusters

AIP Conf. Proc. 1170, 203 (2009); 10.1063/1.3246445

An Oosterhoff Analysis of the Galactic Bulge Field RR Lyrae stars: Implications On Their Absolute Magnitudes

AIP Conf. Proc. 1170, 188 (2009); 10.1063/1.3246439

RR Lyrae Variables in Stellar Systems

AIP Conf. Proc. 1170, 179 (2009); 10.1063/1.3246438

Bayesian Defect Signal Analysis

AIP Conf. Proc. 820, 617 (2006); 10.1063/1.2184584 


\title{
Bayesian Analysis of RR Lyrae Luminosities and Kinematics
}

\author{
Thomas R. Jefferys*, Thomas G. Barnes ${ }^{\dagger}$, Andrei Dambis** and William \\ H. Jefferys \\ ${ }^{*}$ University of Texas at Austin \\ ${ }^{\dagger}$ Department of Astronomy, University of Texas at Austin \\ ${ }^{* *}$ Sternberg Astronomical Institute, Universitetskii pr. 13, \\ Moscow, 119992 Russia \\ $¥$ University of Texas at Austin, and University of Vermont
}

\begin{abstract}
We are using a hierarchical Bayes model to analyze the distances, luminosities, and kinematics of RR Lyrae stars. Our model relates these characteristics to the raw data of proper motions, radial velocities, apparent luminosities and metallicities of each star. A combination of Gibbs and Metropolis-Hastings sampling, using latent variables for the actual velocity and luminosity of each star, is used to draw a sample from the full posterior distribution of these variables, with consideration to identifiability and the properness of the hierarchical model, and draw inferences on the quantities of interest in the usual way. We have applied our model to the large HIPPARCOS database, and we have attempted to include metallicity and period in our model, which has not been done previously.
\end{abstract}

Keywords: RR Lyrae, magnitude, luminosity, HIPPARCOS, Kinematics, Hierarchical Bayes, MCMC

PACS: 02.70.Uu, 98.10.+z, 97.30.Kn, 97.10.Ri

\section{DESCRIPTION OF THE PROBLEM}

RR Lyrae stars are a class of pulsating variable stars. They are readily recognizable from their periods $(0.6 \pm 0.2$ days) and characteristic light curves [1]. These stars have two desirable properties that make them very important in astronomy. The first property is that they are fairly bright, about forty times the brightness of the Sun. Thus, an RR Lyrae star can be seen to fair distances in the Galaxy. The other important property is that the intrinsic visual-band luminosities of RR Lyraes are nearly constant from star to star. This second property is known from studies of RR Lyraes in clusters, where all the stars in the cluster are at the same distance.

These properties make these stars useful as "standard candles" for estimating distances in the universe: We observe an RR Lyrae's apparent luminosity, and from the intrinsic luminosity of the class, use the fact that the apparent luminosity falls off as the square of the distance to work out the distance to the star. Therefore, if an RR Lyrae is embedded in some structure, by finding the distance to it, we then know the distance to the structure in which it is embedded.

RR Lyrae stars have two other properties useful for our study. They have distinctive kinematics, a statistical description of their motions in the galaxy, that imply that they formed in an epoch in galactic history before star formation was confined to the galactic

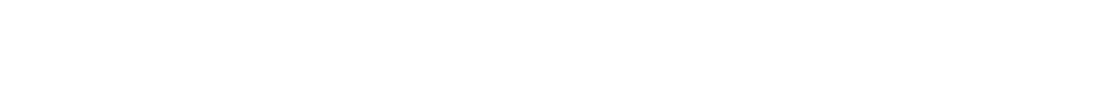


disk. Secondly, they formed over an interval during which the abundances of elements heavier than helium, the metallicity, changed from very low to near solar. Third, they pulsate with a period lasting about a day.

Our goals, therefore, are to determine the characteristic absolute magnitude (proportional to the negative logarithm of luminosity) of these stars as a group, investigate any variation of absolute magnitude with metallicity and period, investigate the cosmic scatter of the magnitude (i.e., variation about the mean unexplained by the covariates), and investigate the kinematics of the stars as a group.

\section{Overview of our Method}

Our raw data for each star include the proper motions $\vec{\mu}$ (vector of angular motion/time unit of the motions of the stars across the sky, in seconds of arc per century), the radial velocity $\rho$ of the motions towards or away from the Sun, in kilometers/second, obtained by observing the doppler shift of spectral lines, and the apparent magnitudes $m$ (proportional to the negative logarithm of the flux) of the star, which is measured with negligible error. For each star, we also have two components of error on the proper motion, $\sigma_{\mu}$, and error on the radial velocity, $\sigma_{\rho}$.

We could infer the the distance to a star from the proper motion if we knew the transverse velocity. The proper motions are related to the transverse velocities (in $\mathrm{km} / \mathrm{sec}$ ) by multiplying the former by the distance $s$ to the star:

$$
s \vec{\mu} \propto \vec{V}^{\perp} .
$$

Similarly, we could infer the distance to that star from the flux and the inverse square law if we knew the luminosity. The inverse square law, mapped to magnitudes is

$$
s=10^{0.2(m-M+5)} .
$$

Extinction, the attenuation of light due to dust between us and the star, is included as a pre-analysis adjustment to $m$ for each star.

We have neither luminosity nor transverse velocity for the stars. However, if we assume that the proper motions and radial velocities are characterized by the same kinematical parameters, we can statistically infer the distances and the magnitudes luminosities of the RR Lyraes through through (2), since we probe these stars in various directions.

\section{THE HIERARCHICAL MODEL}

If for a single star $\vec{\mu}^{o}=\left(\mu_{\alpha}^{o}, \mu_{\delta}^{o}\right)$ are the two components of the observed proper motion vector (in the plane of the sky), and $\rho^{o}$ is the observed radial velocity, we assume

$$
\begin{aligned}
\mu_{\alpha}^{o} \mid V_{\alpha}^{\perp}, s, \sigma_{\mu_{\alpha}} & \sim N\left(V_{\alpha}^{\perp} / s, \sigma_{\mu_{\alpha}}^{2}\right) \\
\mu_{\delta}^{o} \mid V_{\delta}^{\perp}, s, \sigma_{\mu_{\delta}} & \sim N\left(\vec{V}_{\delta}^{\perp} / s, \sigma_{\mu_{\delta}}^{2}\right)
\end{aligned}
$$




$$
\rho^{o} \mid V^{\|}, \sigma_{\rho} \sim N\left(V^{\|}, \sigma_{\rho}^{2}\right)
$$

where $V_{\alpha}^{\perp}, V_{\delta}^{\perp}$ are the $\alpha$ and $\delta$ components of the transverse space velocity, $\vec{V}^{\perp}$, respectively. The variances are measured at the telescope and assumed known with negligible uncertainty. The variables without ' $o$ ' superscripts are the "true" values. The joint distribution over all stars for these three components is our likelihood.

The likelihood defined above constrains the hierarchical model proper, which makes predictions on the proper motions, radial velocities and fluxes of the stars from a set of latent variables. We define the individual absolute magnitude for the star $i$ as $M_{i}=M+U_{i}$, where $M$ is the characteristic absolute magnitude for RR Lyrae stars, and $U_{i}$ is star $i$ 's individual deviation from $M$. Then,

$$
s_{i}=s\left(M, U_{i}\right)=10^{0.2\left(m_{i}-M-U_{i}+5\right)} .
$$

We note that the $s_{i}$ 's are not new parameters; they have no distribution that is not completely inherited from $M$ and the $U_{i}$ 's. The reviewer suggested that it may be possible to include prior information on the space distribution of $s_{i}$ in the anaylsis [2]. We are considering this for future research.

We choose a flat prior on $M$ (we have also tried a somewhat informative prior based on known data, but the results were not significantly different). Evidence from other sources (e.g., studies of RR Lyrae stars in clusters) indicates a cosmic scatter of about 0.15 magnitudes in $M_{i}$. Thus a prior on $U_{i}$ of the form

$$
U_{i} \mid \sigma_{U} \sim N\left(0, \sigma_{U}^{2}=(0.15)^{2}\right)
$$

is appropriate; we explored the effect of this choice (see below).

The priors on the true space velocities, $\vec{V}_{i}$, are obtained by assuming that the velocities of the individual stars are drawn from a three-dimensional multivariate normal distribution with mean $\vec{V}_{\odot}$ (the solar motion) and covariance matrix $W$ (the velocity ellipsoid):

$$
\vec{V}_{i} \mid \vec{V}_{\odot}, W \sim N\left(\vec{V}_{\odot}, W\right) .
$$

We choose a flat prior on $\vec{V}_{\odot}$, and to avoid an improper posterior distribution, a "hierarchical independence Jeffreys prior" on $W$ [3], which for a three-dimensional distribution implies

$$
\pi(W) \propto|I+W|^{-2}
$$

\section{SAMPLING STRATEGY}

We can use Gibbs sampling to sample on $W, \vec{V}_{i}$ and $\vec{V}_{\odot}$. Sampling on $\vec{V}_{i}$ and $\vec{V}_{\odot}$ are straightforward normal distributions with appropriate parameters. Sampling on $W$ is more tricky, as the full conditional is not a closed form. However, we can sample from the full conditional using an importance sampler, using the following proposal:

$$
W^{*} \mid\left\{\vec{V}_{i}\right\}, \vec{V}_{\odot} \sim \operatorname{InverseWishart}(T, d f=N)
$$


where $T=\sum\left(\vec{V}_{i}-\vec{V}_{\odot}\right)\left(\vec{V}_{i}-\vec{V}_{\odot}\right)^{\prime}$, and an importance ratio $P=\left(\left|W^{*}\right| /\left|I+W^{*}\right|\right)^{2}$. Fortunately, in this case the dimension of $W$ is small, $N$ is large, and the proposal $\left|W^{*}\right|$ is typically large, so the acceptance probability is high. (For a more thorough treatment of this problem, see Berger et al. [3].)

In our model, the conditional distributions of $M$ and $U_{i}$ come from the following equality,

$$
p_{N}\left(\vec{\xi}_{i}^{o} \mid \vec{\xi}_{i}, \Lambda_{i}\right)=s_{i}^{2} p_{N}\left(\vec{V}_{i}^{o} \mid \vec{V}_{i}, S_{i}\right)
$$

where $p_{N}(\cdot)$ is a multivariate normal pdf, $\vec{\xi}_{i}^{o}=\left(\mu_{\alpha, i}^{o}, \mu_{\delta, i}^{o}, \rho_{i}^{o}\right)$ and $\vec{\xi}_{i}=\left(\mu_{\alpha, i}, \mu_{\delta, i}, V_{i}^{\|}\right)$ are the observed and true state vector for the star $i, \vec{V}_{i}^{o}=s_{i} \vec{\mu}_{i}^{o}+\rho_{i}^{o} \hat{n}$ is the "observed" space velocity, $\Lambda_{i}=X_{i}+E_{i}$ is the observational covariance matrix and $S_{i}=s_{i}^{2} X_{i}+E_{i}$, both formed from $X_{i}$, the covariance matrix for the observed proper motion vector $\vec{\mu}_{i}^{o}$, and $E_{i}$, the covariance matrix for the observed radial velocity $\rho_{i}^{o}$. The derivation is left as an exercise for the reader, where the identities $\left|\Lambda_{i}\right|=s_{i}^{-2}\left|S_{i}\right|$ and $S_{i}^{-1}=s_{i}^{2} X_{i}^{+}+E_{i}^{+}$will be useful. ${ }^{1}$ Both identities hold because $X_{i}$ and $E_{i}$ are not full rank and live in orthogonal subspaces.

The right-hand side of equation (9) give the conditional distribution of both $M$ and $U_{i}$, products over all $i$ 's in the case of $M$. This conditional must be sampled with randomwalk Metropolis-Hastings for both $M$ and $U_{i}$.

For sampling $M$, we propose $M^{*} \sim N(M, w)$, with an appropriate standard deviation parameter $w$ adjusted for good mixing.

For sampling $U_{i}$ under the informative prior (5), our proposal for a $U_{i}^{*}$ is a locationscale family of a $t$ distribution centered on the origin with an appropriate choice of degrees of freedom and scale adjusted for good mixing. The conditional (9), because of independence in $U_{i}$, may be sampled in parallel.

\section{RESULTS}

Our key astrophysically interesting result is the characteristic absolute magnitude of RR Lyrae stars, $M$. In addition, we are also interested in the solar motion, $\vec{V}_{\odot}$, and the velocity ellipsoid of the galactocentric orbits of the observed RR Lyraes, $W$.

Hawley et al. [4] used a maximum likelihood technique to study this problem using proper motion data from the Annals of the Shanghai Observatory and radial velocity, apparent magnitude, and reddening data from Hemmingway and various sources (all reproduced in their paper, and referred to henceforth as "SHANG"). We compare this to our own results using the same data to demonstrate the improvement using our method, and to our results using the HIPPARCOS data set and improved velocity data [5] from our colleague Andrei Dambis (henceforth, "HIPP”).

Hawley et al. ran cases with a number of subsets of stars, broken down by various criteria. We looked only at the "ab" stars (normal pulsators, $N_{\mathrm{ab}}=143$ ) and the "c" stars (overtone pulsators, $N_{\mathrm{c}}=17$ ), and compared the weighted average of these two groups

\footnotetext{
${ }^{1} A^{+}$denotes the Moore-Penrose matrix inverse of $A$.
} 
in Hawley's study with our own. ${ }^{2}$ Table 1 includes both results for the "ab" and "c" stars analyzed as segregated groups shown, and a weighted average of the two results, but also our own analysis of the HIPP data set as an integrated group $\left(N_{H I P P}=349\right)$. Our results are posterior marginal means and standard deviations.

TABLE 1. Characteristic Absolute Magnitude, $M$, by study

\begin{tabular}{llll}
\hline & Data Set & Star Group & \multicolumn{1}{c}{$M$} \\
\hline \multirow{3}{*}{ This study } & \multirow{2}{*}{ SHANG } & "ab” stars & $0.71 \pm 0.11$ \\
\cline { 3 - 4 } & & "c" stars & $0.72 \pm 0.26$ \\
\cline { 3 - 4 } & & weighted average & $0.71 \pm 0.10$ \\
\cline { 3 - 4 } Hawley et al. & SHANG & all stars & $0.75 \pm 0.07$ \\
\hline \multirow{2}{*}{ SHab" stars } & $0.68 \pm 0.14$ \\
\cline { 3 - 4 } & & "c" stars & $1.01 \pm 0.38$ \\
\cline { 3 - 4 } & & weighted average & $0.72 \pm 0.13$ \\
\hline
\end{tabular}

The sample of "ab" stars agrees within the errors with the analysis of Hawley et al. [4] However, the "c" sample gives a discrepant value of $M$. Their value is anomalously high, compared to our study and to the general consensus on the value of $M$. The best direct measurement of the distance of an RR Lyrae star, by Hubble Space Telescope (Benedict et al. [6]), gives $M=0.61 \pm 0.10$ magnitudes. The results of Skillen et al. [7], using the Surface Brightness method (similar to the method used by Jefferys et al. [8] and reported at ISBA 2000 [8]) give $M=0.65 \pm 0.10$ magnitudes.

Other results of interest are the solar motion $\vec{V}_{\odot}$ relative to the sample and the velocity ellipsoid of the galactocentric orbits $W$.

The solar motion vector informs us of the relative motion of the Sun through the ensemble of RR Lyrae stars in the sample (units are $\mathrm{km} / \mathrm{sec}$ ). Marginal summaries appear in Table 2 , with coordinates in galactic cylindrical coordinates $(\varpi, \theta, z)$; that is, cylindrical coordinates with the origin at the Galactic center.

TABLE 2. Solar motion, $\vec{V}_{\odot}$, by component

\begin{tabular}{lllrrr}
\hline & Data Set & Star Group & $V_{\bar{\sigma}}$ & $V_{\theta}$ & $V_{z}$ \\
\hline \multirow{3}{*}{ This study } & \multirow{2}{*}{ SHANG } & "ab" stars & $-12 \pm 11$ & $-136 \pm 9$ & $-8 \pm 7$ \\
& & "c" stars & $-25 \pm 24$ & $-113 \pm 26$ & $-2 \pm 11$ \\
\cline { 3 - 6 } & weighted average & $-15 \pm 10$ & $-134 \pm 9$ & $-6 \pm 5$ \\
\cline { 3 - 6 } & \multirow{2}{*}{ HIPP } & all stars & $-22.67 \pm 7.97$ & $-141 \pm 5.58$ & $-11.34 \pm 3.96$ \\
\hline \multirow{3}{*}{ Hawley et al. } & \multirow{2}{*}{ SHANG } & "ab" stars & $-10 \pm 13$ & $-155 \pm 12$ & $-9 \pm 8$ \\
& & "c" stars & $-26 \pm 25$ & $-124 \pm 25$ & $-6 \pm 13$ \\
\cline { 3 - 6 } & & weighted average & $-13 \pm 12$ & $-149 \pm 10$ & $-8 \pm 6$ \\
\hline
\end{tabular}

We believe this to be a significant result: using the HIPP data set, we have detected solar motion along both the $V_{\varpi}$ and the $V_{z}$ axes.

\footnotetext{
2 The data of Hawley et al. had some incorrect apparent magnitudes. Reanalysis with corrected data shows that the value of $M$ should be decreased by approximately 0.08 magnitudes. This correction has been applied to Hawley's figures. We have made similar corrections to our own result using SHANG-HIPP needed no such corrections.
} 
The velocity ellipsoid tells us how the galactocentric orbits of the RR Lyrae stars are oriented in a statistical sense, as described by the covariance matrix of the velocities. In galactic cylindrical coordinates $(\varpi, \theta, z)$ the matrix is believed roughly diagonal, with the on-diagonal dispersions decreasing from $\varpi$ to $\theta$ to $z$. (Units are $\mathrm{km} / \mathrm{sec}$ ).

TABLE 3. Velocity ellipsoid, $W$, for normal and overtone pulsators

\begin{tabular}{lllrrr}
\hline & Data Set & Star group & $\sigma_{\bar{\sigma}}$ & $\sigma_{\theta}$ & $\sigma_{z}$ \\
\hline \multirow{3}{*}{ This study } & \multirow{2}{*}{ SHANG } & "ab" stars & $132 \pm 9$ & $108 \pm 7$ & $75 \pm 5$ \\
& & "c" stars & $97 \pm 21$ & $101 \pm 21$ & $44 \pm 10$ \\
\cline { 2 - 6 } & weighted average & $127 \pm 8$ & $107 \pm 7$ & $69 \pm 4$ \\
\cline { 2 - 6 } & HIPP & all stars & $145.60 \pm 5.73$ & $100.16 \pm 4.04$ & $71.10 \pm 2.96$ \\
\hline \multirow{2}{*}{ Hawley et al. } & SHANG & "ab" stars & $150 \pm 59$ & $120 \pm 47$ & $87 \pm 33$ \\
& SHANG & "c" stars & $101 \pm 57$ & 71 & 51 \\
\hline
\end{tabular}

There is a significant difference in the estimated standard deviations between this study and Hawley et al. [4]. Hawley et al. have noted that their use of simplex optimization in the maximum likelihood method made estimating the uncertainties difficult and somewhat arbitrary [9].

Because of the small sample size, in their analysis of these data Hawley et al. [4] did not solve for the full covariance matrix of the velocity ellipsoid for the "c" stars, the overtone pulsator RR Lyraes. Instead, they set the off-diagonal terms to zero, and fixed the ratios for the on-diagonal terms to values given by the reduction of the larger "ab" data. This is why the $\sigma_{\theta}$ and $\sigma_{z}$ do not have standard deviations attached, and no weighted average appears for these results.

\section{DISCUSSION}

\section{Sampling on $U_{i}$}

We find the marginal distribution of the $U_{i}$ to be totally dominated by the prior; as we make changes to the prior, the samples drawn from the posterior change in stepfor instance, varying $\sigma_{U}$ in (5), earlier on, a sample drawn from the posterior has a variance of around this $\sigma_{U}$. From this evidence we conclude that the individual $U_{i}$ 's (and consequently, the cosmic scatter) are unidentified in this model.

This would suggest simply leaving $U_{i}$ out of the model altogether, sampling only on $M$, which would result in a considerable increase in the efficiency of the sampler: We would have only one Metropolis-Hastings step, instead of two. However, J. Berger raised concerns about leaving out the sampling on these variables [10]. Leaving these latent variables in allows us to better estimate the variance on $M$. We find that omitting this set of latent variables did not seem to alter the resulting means significantly, however. 


\section{Unidentifiability of Metallicity and Log-Period}

We had hoped to extend this investigation into the effects of metallicity and period on the individual luminosities of RR Lyraes. The absolute magnitude of a given RR Lyrae star is known to depend on the star's metallicity and period. A commonly used phenomenological relation is:

$$
M_{i}=M+a[F e / H]_{i}+b \log P_{i},
$$

where $M_{i}$ is the absolute magnitude of a certain RR Lyrae star, $[\mathrm{Fe} / \mathrm{H}]_{i}$ is a logarithmic measure of its metallicity (with respect to solar), $P_{i}$ is its period, and $M$ is the characteristic absolute magnitude of RR Lyrae stars. The constants $a$ and $b$ are coefficients relating metallicity and period to deviations from the characteristic absolute magnitude.

We find that actually including metallicity effects in the model produces an unidentified model, as any change in the coefficient $a$ is exactly offset by an appropriate change in the individual velocities of the stars, and so the model is unidentified with respect to $a$, and likely with respect to $b$ as well, although we haven't tested this. Since modeling individual velocities is at the core of our model, we suspect that this is a fundamental limitation of our model.

\section{ACKNOWLEDGMENTS}

This material is based in part upon work by Thomas G. Barnes III while serving at the National Science Foundation. Any opinions, findings, and conclusions or recommendations expressed in this material are those of the authors and do not necessarily reflect the views of the National Science Foundation.

\section{REFERENCES}

1. H. A. Smith, RR Lyrae Stars, Cambridge University Press; Cambridge: New York, NY, 1995, chap. 1,2,4, ISBN 0-521-32180-8.

2. T. Loredo, Private communication (2007).

3. J. O. Berger, W. Strawderman, and D. Tang, Annals of Statistics 33, 606-646 (2005).

4. S. L. Hawley, W. H. Jefferys, T. G. Barnes III, and W. Lai, The Astrophysical Journal 302, 626-631 (1986).

5. T. C. Beers, M. Chiba, Y. Yoshii, I. Platais, R. B. Hanson, B. Fuchs, and S. Rossi, The Astronomical Journal 119, 2866-2881 (2000).

6. G. F. Benedict, B. E. McArthur, L. W. Fredrick, T. E. Harrison, J. Lee, C. L. Slesnick, J. Rhee, R. J. Patterson, E. Nelan, W. H. Jefferys, W. van Altena, P. J. Shelus, O. G. Franz, L. H. Wasserman, P. D. Hemenway, R. L. Duncombe, D. Story, A. L. Whipple, and A. J. Bradley, The Astronomical Journal 123, 473-484 (2002).

7. I. Skillen, J. Fernley, R. Stobie, and R. Jameson, Montly Notices of the Royal Astronomical Society 265, 301 (1993).

8. W. Jefferys, T. Barnes III, R. Rodriguez, J. Berger, and P. Mueller, "Model selection for Cepheid star oscillations," in Bayesian Methods With Applications to Science, Policy and Official Statistics, edited by E. George, Luxemborg: Office for Official Publications of the European Communities, 2001, pp. 243-252.

9. S. L. Hawley, Private communication (2005).

10. J. O. Berger, Private communication (2005). 